\title{
La interpretación textual y la práctica de la investigación cualitativa
}

\author{
Salvador Orlando Alfaro \\ Departamento de Sociologia y Estudios Sociales \\ Universidad de Regina (Canadá)
}

En las ciencias sociales existe una estrategia metodológica que ha cobrado un auge especial en los últimos años denominada generalmente investigación cualitiativa. Existe una especie de consenso académico, al menos en sociología y politología, sobre dicha estrategia, que puede ser explicada del siguiente modo: (a) que lo cualitativo es la "alternativa" a una ciencia social positivista y cuantitativa y (b) que en las condiciones de trabajo y formación actuales, dicha alternativa es más facil de aplicar. Si bien estas dos características deberian ser revisadas con algún detenimiento, desde nuestro punto de vista parten y arriban a conclusiones erróneas. Esto se muestra principalmente cuando se discuten temas vinculados con las estrategias metodológicas particulares, y que es el caso que se abordará a continuación: la lectura del texto sociológico como práctica de la investigación sociológica cualitativa.

Considerando lo anterior, se puede afirmar que la sociología presenta en la actualidad un panorama diferente respecto a la comprensión tradicionalmente aceptada entre lo cuantitativo y la cualitativo. En este panorama, la lectura y comprensión del texto sociológico se transforma en una dimensión clave en la práctica sociológica.

Le Inferprotactón fextual y la próetlea de le investigoción cualitativa 
Con lo anterior se relacionan, al menos, dos prácticas: aquella de leer un texto dentro de un "horizonte" de comprensión y de precomprensión, y la de construir un texto en el proceso de la investigación como comunicación.

La presente reflexión intenta mostrar como para lograr una "adecuada epistemología" de lo cualitativo se deben discutir: (a) las cualidades de los textos creados por la actividad sociológica, (b) el proceso de interpretación que la aludida a la creación implica y (c) el papel de los sujetos que participan en la génesis del documento sociológico.

En el campo de la práctica sociológica enfrentarse a la textualidad no es un fenómeno novedoso. Desde Weber y Simmel, la importancia de las narraciones de los sujetos ha sido un dispositivo fundamental en la comprensión de la realidad. El auge de la investigación cualitativa se ha entendido ( $y$ entiende) como "respuesta" a los enfoques cuantitativos de una sociología positivista (Rossman 1995).

Una de las confusiones más comunes que existe respecto a la investigación cualitativa es la asimilación de las técnicas cualitativas con las estrategias metodológicas. Esta confusión genera muchos problemas de diseño para la investigación de igual dimensión que los que ocasiona realizar una encuesta creyendo que en la aplicación del instrumento se agota el estudio cuantitativo de los procesos sociales. Sumado a esto existe un prejuicio teórico muy arraigado sobre las diferencias entre estrategias cuantitativas y cualitativas tomadas como paradigmas rivales $\mathrm{e}$ inconmensurables.

Si bien una de las características de los enfoques cualitativos de la investigación en ciencias sociales es la íntima relación entre diseño, técnicas, análisis y construcción teórica, que en la práctica invalida una separación radical de las etapas de la misma, es lícito y además conveniente intentar mostrar como se puede reagrupar dichas prácticas según alguna orientación dominante que las asemeje y diferencie.

Renata Tesch (1991) ha esquematizado lo que ella denomina "tipos de investigación", proporcionando una definición de lo que se entiende son los propósitos de los mismos, con la particularidad de que estas últimas son tomadas directamente de la literatura más utili- 
zada en cada temática, lo que la hace aún más interesante. De esta manera se pueden visualizar enfoques diversos para llevar adelante una investigación cualitativa que están agrupados de acuerdo a diversos criterios, entre los cuales predominan el metodológico y el teórico. Dichos enfoques son: (a) Análisis de Contenido (Clásico), (b) Análisis de Contenido (Etnográfico), (c) Análisis del Discurso, (d) Estudios de Documento, historias de vida e historia oral, (e) Etnografía (clásica, holistica, reflexiva), (f) Etnografía (estructural), (g) Etnografía de la Comunicación, (h) Etnometodología, (i) Etnociencia (antropología cognitiva), (j) Análisis de la estructura de eventos y (k) Interaccionismo simbólico.

La pluralidad es innegable. En cada uno de estos enfoques las unidades de registros y de análisis cambian o son entendidas de forma diversa, el lugar de la natural textualidad de lo cualitativo es diferente, las técnicas pueden o no ser las mismas, pero por lo general unas se adecuan mas que otras y los puntos de partida teóricos o estos son los que se transforman radicalmente. El significado de las formas de crear, reproducir e interpretar la acción en tanto narración se modifica y varía, aparecen así desde la correlacion estadística hasta la semiótica como "instrumentos" para la interpretación.

La enumeración realizada arriba, autoriza sólo a constatar dos elementos útiles para los objetivos de esta reflexión: por un lado saber que existen diferentes técnicas y diversas formas de hacer investigación cualitativa y por otro que a través de una simple enumeración se puede observar que la relación cuatitativo/cualitativo no es, como se afirma, necesariamente aporética. Para ilustrar este ultimo punto, parece conveniente resumir lo que Ray Pawson (1993) ha denominado "fases de la relación cualitaivo/cuantitativo".

La primera fase se caracteriza por permanecer dentro del puritanismo metodológico, donde la actividad de investigación podía relacionarse dicotómicamente con uno u otro enfoque. Se relaciona con la así llamada "guerras de los paradigmas" metodológicos y las discusiones "metrocentradas".

La segunda fase hace referencia al surgimiento de un pluralismo pragmático como reacción a la metodolatría, teniendo a las estrategias de "triangulación" como fundamento operativo. Es una fase centrada 
en la elaboración y resolución de problemas donde se acepta que existen diversas vías metodológicas para lograr ese objetivo.

La tercera fase, que aún hoy se desarrolla, consiste en la superación de la relación cualitativo/cuantitativo desde un punto de vista docotómico diluyendo un tratamiento aporético de la misma. Centrada en lo relacional apunta a que la discusión se desplace hacia la mejor articulación entre estrategias que puedan dar cuenta de la conexión entre mecanismos, contextos y agentes.

Finalmente, este apartado respalda la posibilidad de introducirse, sin entendidos equivocados, en algunas discusiones sobre el lugar de la "textualidad" en la investigación cualitativa. Como guía de la discussion se ha optado por ordenar la argumentación de la siguiente manera: en primer lugar, desde un punto de vista epistemológico se intentará aclarar la relación entre datos, narración e interpretación, haciendo especial referencia a la conexión entre, manifestaciones, proposiciones e interpretación; luego desde la misma perspectiva se aclara cómo se puede conectar datos y documentos sociológico. En segundo lugar, desde un cruce entre filosofía y sociología se tratará de explicar la interpretación como práctica sociológica. Por ultimo, en tercer lugar, se deducirán algunas conclusions epistemológicas y metodológicas que permitan re-pensar el lugar de la interpretación en la investigación cualitativa.

Uno de los problemas más interesantes de la aceptación de la estructura narrativa de la investigación cualitativa es el de redefinir el lugar y la noción de dato que ella implica. En estrecha conexión con esto se puede identificar la tarea de construir un texto como resultado de la estrategia cualitativa. En lo que sigue se pretende abordar estas dos temáticas intentando contextualizarlas en el marco actual de los debates en la filosofía de las ciencias social (Sayer 1992).

\section{Manifestaciones, proposiciones e interpretación}

La investigación cualitativa enfrenta a quienes practican la investigación en el campo de lo social con la necesidad de realizar un análisis de la noción de dato. En este contexto discursivo se encuentran algunos elementos para aclarar la función de los datos como garantías de 
las interpretaciones y su conexión con la noción de evidencia. Desde una perspectiva clásica se puede afirmar que los datos son la manifestación verbal o escrita de la observación que se refiere simbólicamente a una unidad determinada del fenómeno observado. Lejos de emprender una crítica sistemática de la mencionda noción, aquí se intenta enfatizar, que es ella misma la que establece las bases para comprender como datos tienen siempre una estructura relacional. Esto quiere decir que su composición es una conexión explícita entre afirmación, observación y fenómeno que no se puede reducir a ninguno de estos tres momentos del conocimiento social.

En la investigación cualitativa se puede ver claramente como la base empírica de la investigación tiene la forma de una estructura diferenciada en diversos "niveles" de afirmaciones.

Estos niveles contienen como elementos diferentes proposiciones que se diferencian de acuerdo a: su contenido, su puesto en la lectura (que origina el tipo de interpretación) y según la distancia con la materialidad de la observación.

De este modo la investigación cualitativa supone el manejo de afiramciones producidas por la narración del mismo fenómẹno, que implican una primera lectura de la realidad y que tienen una aproximación mayor con lo concreto entendido como materialidad que responde a la pregunta: ¿qué es eso? Se puede visualizar que la investigación cualitativa involucra proposiciones desciptivas que se ubican como puente entre observación y lectura segunda y que comparten en algún modo ambos polos de la relación concreto-abstracto. Finalmente se pueden identificar afirmaciones interpretativas que constituyen el comienzo de la segunda lectura y que son más abstractos que los otros niveles.

(1) narración-manifestación primera lectura_concreto

(2) proposición descriptiva

(3) afirmación interpretativa lectura segunda__ abstracto

Es evidente que la relacionalidad de los datos se manifiesta en la estratificación de los enunciados que contienen las observaciones. En esta estratificación es claro que las posiciones entre sujetos son intercambiables y que las enunciaciones son efectuadas siempre en tercera persona desde el punto de vista de la pragmática del lenguaje. 
En este sentido, se puede aclarar como la base empírica de la investigación cualitativa aparece como garantía de interpretación. En el caso que la pretensión de validez de la interpretación sea puesta en duda, se responde señalando que el nivel de estratificación que ha sido objeto de cuestionamiento y se reconstruye paso a paso los componentes de la estructura con el objeto de develar la base que sostiene la enunciacion criticada.

Para evitar un círculo que vuela a remitir la discusión a la afirmación puesta en duda se debe poder señalar los mecanismos que se han utilizado en el pasaje de la narración a la proposición descriptiva. Emerge de esta manera que los datos que tienen una función de garantía de la interpretación entendiendo por esta a la argumentación racional que conecta la racionalidad de los datos con la forma estructural de estos tienen y que permite reconstruir la relación misma como los momentos de conexión entre afirmaciones, observaciones y fenómenos.

\section{Dato y documento sociológico}

Por otro lado, aparece el hecho particular que en la investigación cualitativa los datos no se cuentan sino se "re-cuentan", y así, emerge el problema de afrontar una teoría del texto sociológico en tanto documento construído por la "acción sociológica". Los datos producidos por la investigación cualitativa tienen una dependencia mayor de la narración y en ello nace la objetivación de la interpretación como documento sociológico.

Una pregunta fundamental nace aquí: ¿Cómo se puede analizar la relación entre registro, escritura e intepretación de un texto sociológico? En una primera mirada se propone la utilización del término "documento" para designar el resultado escrito construído temporalmente entre diálogo efectivo e interpretación segunda. El texto sociológico proviene de la reconstrucción del documento como manifestación de diálogo. Para llegar a la construcción del documento, la práctica sociológica sigue el camino que va de la comunicación y se mueve sucesivamente a la narración, el registro, la escritura, la interpretación y la reflexividad arribando nuevamente a la comunicación (Coulon 1995). 

(1) comunicación
(3) registro
(5) intrepretación
(2) narración
(4) escritura
(6) reflexividad

En la investigación de las ciencias sociales aparece como primer movimiento de la comprensión la necesidad de captar el horizonte compartido pre-reflexivo de la acción. El juego entre la experiencia de construcción, documento y sentido de la acción implica un re-leer el horizonte de sentido compartido partiendo de la diversidad. Por lo tanto, la meta inmediata de la acción sociológica es tomarse a si misma como acción humana. En esta dirección aparecen dos momentos de la dialéctica de la comprensión:

(a) El momento del auto-reconocimiento de aquel que hace la búsqueda como sujeto inmerso en una intersubjetividad (Melucci 1996),

(b) El entender que la acción sociológica es un modo particular de acción sobrepuesta a las reglas compartidas de la vida cotidiana en tanto acción científica.

En este marco de experiencia (y en un primer momento) la pregunta sociólogica no es otra que la pregunta por una fenomenología de la vida cotidiana que permita iluminar el juego de comprensión y precomprensión de la relación intersubjetiva. $\mathrm{Tal}$ fenomenología puede entenderse como reconstrucción racional del mundo de la vida donde se encuentran las raíces de un sujeto-en-el-mundo.

Así, los habitantes de esta región de la experiencia son los productos de la práctica cotidiana de los agentes sociales. El sentido que emerge en la relación pregunta, respuesta e interpretación, manifiesta la acción humana como co-contrucción. Pero no todo es construido, el lenguaje por el cual los objetos llegan a ser interpretados se concecta con la tradición en tanto herencia teórica a la cual pertenece quien quiere comprender, y como "forma de vida" que siempre esta ya presente entre dos personas que se quieren entender.

El movimiento que hay en la actividad de traducir una narración es un movimiento cargado de sentido en sí mismo. El texto sociológico en su intención de captar sentido de la acción encuentra al final su propio punto de partida: la posibilidad de la pluralización de los mundos. El movimiento de la comprensión no se detiene, solamente puede ser detenido como objeto de una decisión del lector. La pequeña 
historia entre sujetos que emerge en la relación sociológica puede ser narrada de nuevo como otra experiencia y por eso necesita ser detenida en el diálogo y el acuerdo. En este marco, "el documento sociológico deviene evidencia de un horizonte de sentido como decisión dialógica con la intención de representar su significatividad" (Patton 2002: 43). Desde aquí se puede apreciar como emerge la centralidad de la práctica sociológica en la tarea de construir una narración compartida de la realidad social.

\section{IV}

En conexión con lo que se ha expuesto, se puede relacionar ahora, dos prácticas: aquella de leer un texto dentro de un "horizonte" de comprensión y de precomprensión, y la de construir un "texto" en la dinámica de la investigación como comunicación. Sin duda esta conexión tiene bastante presupuestos por develar y aclarar, pero se pueden considerar como punto de partida, para la reflexión, dos experiencias: la de lectura y la de comunicación (May 2002: 58). En este contexto se plantea una pregunta como esquema para la búsqueda propuesta: Se puede utilizar el enfoque filosófico sobre la lectura de un texto para iluminar la inscripción, escritura e interpretación de un texto sociológico?

La misma pregunta es ya una interpretación de la actividad de la lectura en el contexto de una hermenéutica (May 2002: 69), en la cual la principal finalidad es la comprensión y construcción del pensamiento, es el hacer saber el sentido de una relación entre escritura y lectura. Interpretación que parte de la aceptación de que leer es proveer de sentido al texto desde un sujeto que es el lector y desde otro que es un co-lector. Esta perspectiva hace pensar que una conexión entre la "lectura filosófica" y la práctica sociológica posible.

La finalidad aquí, será subrayar los aspectos de la práctica sociológica que aparecen como semejantes a la práctica filosófica de leer un texto. También se intentará, originar en la práctica interpretativa de la hermenéutica (que hace reflexión de la relación, texto, autor, lector su medio específico para pensar y pensarse) una reflexión sobre la práctica sociológica.

Desde este punto de vista, la sociología (re) toma la acción a través de la palabra, haciendo una comparación con la afirmación bien cono- 
cida de Ricoeur "La palabra dará que pensar". La acción es así un símbolo que produce una llamada a la interpretación. Desde esta perspectiva, la sociología no se puede comprender sí no se acepta que todos sus actos son actos de comunicación e informes inter-subjetivos. La acción no es el lenguaje, pero solamente se puede comprender en el especial medio que anida en el lenguaje, porque esa práctica sociológica se piensa como una práctica comunicativa y toma como punto de partida el diálogo entre dos sujetos produciendo sentido a esa misma acción. Por consiguiente, interpretar una acción es una práctica de lectura que pone en relación acciones, lenguaje y vida cotidiana del sujeto. Fenoménicamente esta práctica aparece como narración, conciencia discursiva y como documento sociológico, estos tres elementos aparecen como la primera objetivacion del diálogo sociológico (Myerson 1994). El diálogo sociológico implica la relación entre dos o más sujetos donde una o algunas de ellas entran al intercambio en posición objetivamente y tienen capacidad en tanto seres humanos de pasar a una actitud reflexiva por la cual el diálogo mismo se hace posible.

La investigadora o investigador social como sujeto perteneciente a un mundo de la vida, tiene la cualidad de poder "darse cuenta" de sus acciones en la forma de una narración. Esta capacidad de dar respuesta, esta "responsabilidad" (accountability) implica el poder de expresar e interpretar la acción misma, así el actor-se re-presenta en su vivir cotidiano desde el punto de vista de sí mismo como sujeto de la acción. La capacida de narrar es un hecho que surge de la conciencia discursiva como cualidad de los seres humanos de transformar la práctica en la palabra, la actividad en el lenguaje. La sociología provee como resultado del diálogo un documento que como reinterpretación re-construye la narración y cristaliza la conciencia discursiva del sujeto, por lo tanto, la acción sociológica es una acción interpretativa, y una segunda lectura hecha por el co-autor de un texto.

La comprensión sociológica se pone en marcha como resultado de una interpretación del documento que genera, tomando como supuestos los rasgos "ontológicos" que hacen comprender el enlace entre constitución del sí-mismo en el diálogo con otro sí-mismo, transformándose así, en dicho diálogo, la interpretación misma.

Una hermenéutica de la acción se puede entender como una experiencia de lectura del espacio que está entre el lenguaje y la acción,

La inferpretación foxfual y la préctica de lo investigación cualitativa 
espacio que se constituye en el diálogo que posibilita pensar a la sociedad de modo diferente. La pregunta específica de la sociología es aquella que hace de la relación social un hecho de sentido y que está orientado a comprender este sentido construido. La hermenéutica, entonces parece ser la actividad de evidenciar el sentido de la relación social en el contexto de un relato entre dos sujetos, el o la analista social que intenta comprender y otro sujeto que sabe qué cosa es el sentido de su actividad en el mundo de la vida. Emerge por esta vía el "informe" del diálogo aludido. Este informe se cristaliza en el escrito sociológico que el sociólogo o la socióloga tiene como regsitro. Dicho escrito aparentemente sin sentido, parece ser al mismo tiempo una lectura que provee una primera interpretación, y así, una primera imposición de la relación entre sujeto que quiere conocer y sujeto que conoce. Se genera de este modo, otra interpretación efectivizada como necesidad del acto de comprensión.

La interpretación sociológica produce otra acción que se manifiesta como texto, vale decir, como objeto de una lectura que cosntituye un contexto de la relación y de la misma primera interpretación. Así, emerge un texto que hace de (co) texto y que necesita de ser interpretado en el continuo de dos historias, las historias de dos sujetos, que en la propia relación construye su interpretación. La construcción de sentido parece ser una co-construcción de un texto que se manifiesta como red entre pensamiento, historia e interpretación.

La co-construcción es creada como dialéctica entre palabra, ausencia y silencio. Palabra como pregunta por la acción, ausencia como relación sobre la cual se necesita el sentido, y silencio como doble juego de lo no dicho y aquello que es la respuesta. Se puede entender como la reconstrucción de la acción como una construcción de sentido respecto al diálogo suprimido y en el diálogo ahora escrito y reinterpretado.

La interpretación se entrecruza con la traducción en la acción comunicativa, "los otros" que hablan sobre su mundo de la vida son sujetos de una interpretación que parece ser una traducción del sentido de la acción en el horizonte comprensivo compartido. Y que al mismo tiempo necesita de un desplazamiento, de una nueva re-interpretación, en esta ocasión sobre la forma de narración construida como objeto más allá del proceso de pregunta-respuesta. Por lo que, el mo- 
verse entre sujeto-sujeto (el diálogo) por un objeto (texto) que cristaliza pero no finaliza ni agota el sentido, hace aparecer la racionalidad que supone la comunicación. Por esto, el diálogo se puede entender como proceso de conocimiento y de interpretación de la acción que se busca comprender.

Finalmente, entonces, el texto es evidencia inter-subjetiva del diálogo como proceso de conocimiento y de construcción compartida del sentido de la acción, evidencia de una experiencia de re-lectura entre dos sujetos con dos narraciones que surgen desde un mismo horizonte de comprensión. El diálogo hace reingresar el sentido en el contexto intersubjetivo como pensamiento y como racionalidad. Como pensamiento por sus características en tanto "juego del lenguaje" que hace volver a los sujetos objetos de sí mismos. Como racionalidad es el resultado conocido del proceso mismo de re-conocer el texto de un mundo de la vida.

\section{V}

Entendiendo, del modo arriba expuesto, el lugar de lo textual se pueden deducir algunas consecuencias de orden metodológico para el diseño y análisis de las investigaciones cualitativas.

En primer lugar se puede pensar algunas "condiciones de entrada" a la elaboración de diseños cualitativos. De esta manera, para poder introducirse a la ejecución de un diseño cualitativo se deben tener en cuenta los siguientes supuestos:

(1) La asimetría estructural, o major las diferencias de posición y condición (en el sentido de Bourdieu 1990) entre el investigador y los sujetos de la investigación.

(2) El rasgo fundamental del investigador social que qua sujeto comparte el conocimiento de lo social con el sujeto que coconstruye el documento sociológico con él.

(3) La necesaria "descentralización" del investigador, es decir, el abandono consciente y explícito del centro de las relaciones que genera la actividad de investigación.

(4) La importancia de planificar dispositivos de "socio-análisis" de las practices involucradas en la acción sociológica, lo que implica la 
incorporación de búsquedas tendientes principalemente a reflexionar sobre la reflexividad de la misma.

Por otro lado, si se entiende la interpretación y construcción del modo que se ha expuesto se puede conocer mejor que tenemos cuando hacemos análisis en la investigación cualitativa:

Por un lado tenemos registros progresivos de lo observado, es decir, tenemos objetos que se ubican en algún punto de una espiral compuesta y re-compuesta por realidad y teoría formando un texto. Si bien, el grado de constructibilidad de estos objetos depende de nuestro enfoque teórico y de los supuestos ontológicos y epistemológicos al cual este responde, particularmente se puede indentificar una estratificación básica que involucra la relación entre:

(1) narración - manifestación primera lectura

(2) proposición descriptiva

(3) afirmación interpretativa lectura segunda abstracto

Por otro lado, tenemos un juego de interpretaciones que proporcionan los sujetos de la investigación más cerca del análisis que pretende nuestra teoria. Aquí se debe tener siempre presente que tanto investigador como investigado son considerados como sujetos "portadores" de dichas interpretaciones. Esta relación se da en el marco del movimiento entre:

(1) comunicación (3) registro (5) interpretación

(2) narración (4) escritura (6) reflexividad

Finalmente, se puede iluminar lo que hacemos cuando analizamos:

En primer lugar, reconstruimos lo que vemos en un equilibrio entre pluralidad y singularidad a partir de un proceso de comprensión en el marco de una dialéctica de pregunta-respuestas.

En segundo lugar, subsumimos las reconstrucciones en una red de interpretaciones. Este es el punto de partida del contínuo que va de la comunicación a la reflexividad. Luego, como subsumir incluye una tarea de inclusión, procedemos a corregir pero no la realidad sino las interpretaciones. Este corregir es sugerido por un proceso que incluye (a) un análisis crítico de las condiciones de análisis que hemos puesto, (b) de las precondiciones de visibilidad de lo analizado y (c) 
por lo que se ha denominado aquí construcción del documento sociológico. Por esta vía, (a) se refiere a una redefinición de los modos de "definir" los registros y los datos; (b) implica un ajuste de la coherencia entre preguntas, técnicas e interpretaciones y (c) involucra revisar la relación entre texto, práctica y sentido.

Por todo esto, reconstruir, subsumir y corregir devienen en argumentar, esto al menos implica:

(A) Escribir, es decir construir un Texto, sujeto de intersubjetividad; $y$

(B) Dar razones sobre la particularidad de nuestras interpretaciones apelando a la estructura relacional de los datos tal como arriba se ha expuesto.

Entendida de esta manera la tarea sociológica de interpretación parece, al menos de forma provisoria, poder ligarse con la interpretación del texto que supone la investigación cualitativa. La práctica sociológica es una interpretación segunda que se configura en un diálogo que fundamentalmente se objetiva en el documento sociológico. Por esta vía, lo cualitativo del texto sociológico se presenta como característica promaria de conocimiento sobre lo social.

Es justamente en el centro de esta complejidad que se pone en movimiento la relación diálogo, interpretación y práctica sociológica, donde filosofía y sociología se entrecruzan una vez más. La posición de los sujetos en la co-construcción del documento sociológico señala en la dirección de recuperar lo que hay de humano en la práctica reflexiva de la acción sociológica.

\section{REFERENCIAS}

Bourdieu, P. (1990) In other words: essays towards a reflexive sociology. Cambridge: Polity Press.

Coulon, A. (1995) Ethnomethodology. Thousand Oak, CA: Sage Publications.

May, T. (2002) Qualitative Research in Action. Thousand Oak, CA: Sage Publications.

Melucci, A. (1996) Challenging Codes. Collective Action in Information Age. Cambridge: Univeristy of Cambridge Press. 
Myerson, G. (1994) Rethoric, Reason and Society. Londres: Sage.

Outhwaite, W. (1987) New Philosopby of Social Science. Nueva York: St. Martin's Press.

Patton, M. (2002) Qualitative Research and Evaluation Methods. Thousand Oak, CA: Sage Publications.

Pawson, R. (1994) "Quality and Quantity, Agency and Structure, Mechanism and Context, Dons and Cons" Nuffield College 1993. Rossman, G. (1995) Designing Qualitative Research. Thousand Oaks: Sage.

Sayer, A. (1992) Method in Social Science. Londres: Routledge.

Tesch, R. (1991) "Software for Qualitative Reserachers: Analysis Needs and Program Capabilities", Lee, T. y Feelding, S. Using Computers in Qualitative Research. Londres: Sage. 16-38. 